\title{
Experimental hypoxic brain damage
}

\author{
J. B. BRIERLEY \\ From the Medical Research Council Laboratories, Carshalton, Surrey
}

The majority of hypoxic episodes that result in histologically proven damage in the human brain cannot be adequately defined in physiological terms. They are usually accidents so that basic information such as the precise duration of a cardiac arrest or the blood pressure and heart rate during a period of severe hypotension is very rarely available. In such cases, neuropathological descriptions, however exhaustive, may well explain the final neuropsychiatric status of the patient but can at best indicate only tentatively the nature of the episode itself.

The experimental approach is justified if it can indicate whether damage of a particular type in neurones and in white matter is or is not a direct consequence of a particular hypoxic stress adequately delineated in physiological terms.

At the outset it must be recalled that the energy for the normal functioning of the central nervous system is derived from the oxidative metabolism of glucose. A deficiency of oxygen or glucose will impair function and if severe and protracted enough will lead to irreversible brain damage. Interruption of the oxygen supply produces the most rapid impairment of brain function. Thus consciousness is lost about $10 \mathrm{sec}$ after circulatory arrest. Abrupt anoxia exemplified by inhalation of an inert gas or sudden decompression to an altitude above 50000 $\mathrm{ft}$ leads to loss of consciousness after a slightly longer interval (17-20 sec). This rapid loss of consciousness in instances of profound hypoxia may well be responsible for the widely held view that enduring brain damage may begin soon after consciousness is lost.

\section{Types of hypoxia}

Before considering the relationships between the known neuropathological patterns in the human brain that are ascribed to hypoxia and their apparent counterparts in the brains of experimental animals, it will be useful to classify the several types of hypoxia. However, it will be shown that there is no justification for the assumption that each type of hypoxia can, per se, give rise to brain damage. The original classification of Barcroft (1925) must be modified in the light of subsequent information from human and experimental animals sources as follows:

\section{ISCHAEMIC}

Blood flow is arrested in the brain as a whole or in the territory of a single artery.

\section{OLIGAEMIC}

A reduction in blood flow in the brain as a whole or within the territory of a single artery may occur as a result of a greatly reduced cardiac output or major systemic hypotension from any cause.

\section{ANOXIC}

The arterial oxygen tension is $\mathbf{0 m m ~} \mathbf{~ H g}$. It occurs if inert gases are inhaled, if there is total obstruction of the upper respiratory tract or in the event of sudden exposure to an altitude greater than $50000 \mathrm{ft}$ (the combined tensions of water vapour and carbon dioxide within the pulmonary alveoli then exceed the ambient pressure and no oxygen can enter the lungs).

\section{HYPOXIC}

There is some reduction in a $\mathrm{pO}_{2}$ short of anoxaemia. This occurs in chronic pulmonary disease and in congestive heart disease; when the inspired oxygen is diluted by an inert gas (as in some anaesthetic accidents) and also in exposures to altitudes less than $50000 \mathrm{ft}$.

\section{ANAEMIC}

There is some reduction in the amount of circulating haemoglobin available for combination with oxygen. It can occur after severe haemorrhage, in severe hypochromic anaemia but the commonest apparent cause of anaemic brain damage is carbon monoxide intoxication.

\section{Histotoxic}

This implies the poisoning of oxidative enzymes within neuronal mitochondria. Cyanide and azide are examples.

\section{HYPOGLYCAEMIA}

A deficiency of the principal substrate, glucose, per 
$s e$ can also give rise to ischaemic cell change even if the level of arterial oxygenation is normal.

The previous contributors to this section of the Symposium have defined the nature and time course of ischaemic cell change and have pointed out that it is the principal neuronal response to all types of hypoxia in the brains of rodents as well as in those of primates including man. In this survey of the brain damage attributable to hypoxia in all its forms, only the patterns of distribution of ischaemic cell change will be considered with emphasis on the contributions from experimental studies.

\section{Ischaemic}

Arrest of circulation within a single brain artery results in an infarct which can range in size from the 'total territory' in an anatomical sense to a small volume of tissue close to the point of arterial occlusion. Where the cortex of cerebrum or cerebellum is concerned the extent of infarction is determined by the level of systemic blood pressure at and after the instant of occlusion and, in particular, by the functional efficiency of the leptomeningeal vessels that anastomose with the cortical branches of neighbouring arteries. If these anastomotic systems and the major arteries in the neck and the circle of Willis are normal, the cortical infarct will be small. If one or both are the site of occlusive vascular disease, the infarct will be larger.

It must be borne in mind that the basal ganglia and the internal capsule, in particular, are supplied by end-arteries (penetrating or ganglionic branches of the major cerebral arteries). Occlusion of an arterial trunk proximal to the ganglionic branches produces an infarct in these deeply placed regions of grey and white matter even in the healthy experimental primate. Evidently the retrograde flow of blood from leptomeningeal anastomoses into the arterial stem may never enter all its ganglionic branches or, if it does so, it may be too little and too late to avert irreversible tissue damage. Thus, for example, division of the middle cerebral artery close to its origin from the internal carotid artery in the baboon leaves the sensory and motor cortex intact and cortical infarction is confined to some portion of the insula. A variable hemiparesis involves only the contralateral face and upper limb and its neuropathological basis has been shown to lie entirely within white matter, ie, in the genu and supralentiform portions of the internal capsule, where after a survival of three years there is a sharply circumscribed cystic infarct (Symon and Brierley, 1976). The limited neurological deficit and the small, deeply placed infarct that follow division of the middle cerebral artery in a healthy experimental primate are sharp reminders that such 'models' cannot provide two of the most important factors in the aetiology of 'stroke' in man. These are some impair- $\Rightarrow$ ment in cardiac function (leading to some reduction $\stackrel{5}{9}$ in cerebral blood flow) and some degree of occlusive vascular disease. These factors, singly or together, account for the extension of the infarct into the $\frac{\bar{\omega}}{\frac{}{5}}$ centrum semi-ovale and even into the whole of the $\stackrel{\mathbb{D}}{\square}$ anatomical cortical territory. It follows, that in the human brain, ischaemic necrosis in some portion of $\vec{\theta}$ an arterial territory can seldom be explained $\stackrel{\circ}{-}$ satisfactorily without careful examination of the $\vec{\omega}$ myocardium, the coronary arteries and the major arteries of the neck and brain.

Overall or global arrest of the brain circulation ${ }_{\omega}$ leads to a loss of consciousness in eight to $10 \mathrm{sec}$ and the EEG is isoelectric a few seconds later. Respira- $\overrightarrow{-}$ tion fails at about the same time while the heart may continue to beat for a matter of minutes. Neuro- $\stackrel{\infty}{-}$ pathological descriptions of the consequences of음 circulatory arrest (including 35 personal cases) pro-- vide the best examples of the involvement of the 'selectively vulnerable' regions of the brain in? hypoxia. Frequently, little of the cerebral cortex is normal but damage is usually greater in the posterior half of each cerebral hemisphere, in the floors $8 f$ sulci rather than over the crests of gyri and in third, fifth and sixth layers rather than in the secoød and fourth. Certain portions of the hippocamp (zones h.1-Sommer sector-and h.3-5,-endfolium) are vulnerable as are the Purkinje cells of theo cerebellum. Many sensory nuclei in the brain stem are vulnerable in the infant and young child (Ranck $\overrightarrow{0}$ and Windle, 1959; Brierley, 1965, 1976).

Where circulatory arrest has been studied in the? experimental animal, it is important to recognize that earlier studies were concerned to define the maximum period of arrest of the cerebral circulation:beyond which some degree of irreversible brain 3 . damage would occur. Attempts to define such ai 'threshold' have been reviewed by Hoff et al (1945), Meyer (1963) and Brierley (1976). The generab conclusion from these studies has been stated by? Schneider (1963) as follows: 'A complete revivato without neurological or histological damage cannot be brought about after a complete stop of brainos circulation of more than four to five min duration'. N

In contrast to the experiments summarized aboven certain recent studies have attempted to define of much greater period of circulatory arrest after which there can be some evidence of recovery in at least at neurophysiological sense and histological examination can show that some parts of the brain are normal ${ }_{-}$ Thus Hossmann and Sato (1970) claimed tha $\overline{5}$ ... . unequivocal signs of neuronal recovery can be्ष detected after complete ischaemia of more than one 
hour's duration'. Hirsch et al (1975) failed to confirm these results and attributed 'recovery' after such protracted ischaemia in the experiments of Hossmann and Sato (1970 and subsequent studies) to the protective effects of anaesthesia and the progressive fall in temperature that must occur in the isolated head during such periods of time.

It must be emphasized that experimental studies of the effects of circulatory arrest (or any other form of hypoxia) on the brain, whether directed towards the definition of a 'threshold' for a particular hypoxic stress or to the capacity for recovery after an extended period of the same stress, have clinical relevance only if spontaneous respiration has been resumed in the unmedicated animal, and detailed neurological assessments, together with serial recordings of the EEG, have been made during an adequate period of survival. All these are essential for a meaningful appraisal of 'recovery'. Finally, after in-vivo perfusion-fixation of the brain, neuropathological examination of the brain must be comprehensive. Unfortunately clinico-pathological studies according to such standards have not yet been reported in support of the claim that 'recovery' of the central nervous system can occur after periods of circulatory arrest far in excess of those hitherto accepted as 'critical' where the inception of minimal brain damage is concerned.

\section{Oligaemic hypoxia}

A reduction of blood flow in a single artery of the human brain is usually due to a combination of systemic hypotension and occlusive disease in the vessel itself. If flow is sufficiently impaired the outcone will be an infarct involving grey and white matter. Such a local reduction in flow can only be inferred if thrombosis and embolism can be excluded. There are, as yet, no experimental models of this particular situation.

Global oligaemia implies some reduction in the overall flow of blood through the brain. Experimental studies in the Rhesus monkey have shown that, if arterial oxygenation remains normal, cerebral perfusion pressure (mean arterial blood pressure - venous sinus pressure) must be reduced to $25 \mathrm{~mm} \mathrm{Hg}$ for at least $15 \mathrm{~min}$ before brain damage is produced (Brierley et al, 1969; Meldrum and Brierley, 1969). However, it was only possible to damage the brain if the profound hypotension was continued beyond the point of apnoea when mechanical ventilation was required in order to maintain a normal arterial oxygen tension. These experiments clearly demonstrated that in the healthy spontaneously breathing primate, global oligaemia per se is unlikely to lead to brain damage if respiration does not fail. In these monkeys, typical ischaemic neuronal alterations were not evenly distributed in the cerebral cortex but were restricted to the arterial boundary zones of the cortex of the cerebrum and also of the cerebellum. They were variable in the basal ganglia. The pysiological basis of lesions along arterial boundary zones has been discussed by Zülch and Behrend (1961) and by Meldrum and Brierley (1971). When perfusion pressure falls below $45-50 \mathrm{~mm} \mathrm{Hg}$ the capacity of the vascular bed to maintain a constant cerebral blood flow (autoregulation) is lost (there is then maximum vasodilatation) and flow becomes directly dependent upon perfusion pressure. The reduction in flow is greatest in the vessels most remote from the arterial stem, ie, at the boundary of each arterial territory.

In the clinical situation, a reduction in brain perfusion pressure while arterial oxygenation remains normal is virtually confined to the technique of hypotensive anaesthesia with controlled ventilation and then only when perfusion pressure through the brain is lowered by excessive head-up tilt (Brierley and Cooper, 1962). The two additional factors that may result in brain damage after a period of relatively moderate hypotension are some degree of hypoxaemia and some element of occlusive disease in the extra- and/or intracranial arteries. The frequency of these two factors in addition to the reduction in brain blood flow due to the initial systemic hypotension is largely responsible for the fact that ischaemic damage along arterial boundary zones of the cortex of cerebrum and cerebellum is the commonest neuropathological outcome of hypoxia in all its forms. It is important to appreciate that no experimental model permitting the introduction and control of oligaemia, hypoxaemia and partial vascular occlusion is yet available.

Previous contributors to this section of the Symposium have emphasized that this 'boundary zone' pattern of brain damage can only be identified if blocks for histological examination are selected with an awareness of the anatomical distribution of the cortical arteries of cerebrum and cerebellum.

\section{Anoxic}

Anoxia, induced by breathing pure nitrogen, has been studied in human volunteers by Gastaut $e t$ al (1961) and Ernsting (1963). After a few seconds the EEG shows low voltage activity at 11 to $13 \mathrm{c} / \mathrm{s}$ and consciousness is lost at 17 to $20 \mathrm{sec}$. In experimental animals, longer periods of nitrogen breathing lead, after an initial hyperventilation, to slowing of respiration, bradycardia and a falling blood pressure. Apnoea occurs at about the third minute while blood pressure is still appreciable $(5-20 \mathrm{~mm} \mathrm{Hg})$ at 
the fifth minute (Swann and Brucer, 1949). In the Rhesus monkey, the responses to nitrogen breathing are similar and if mechanical ventilation is begun soon after the 'last breath', the blood pressure rises, spontaneous respiration is resumed and the EEG, previously isoelectric, returns to normal. Subsequent neuropathological examination reveals no brain damage (Brierley and Meldrum, unpublished observations). Evidently the period of anoxaemia and of secondary circulatory impairment is too brief to lead to ischaemic neuronal alterations so that it must be concluded that pure anoxic anoxia cannot produce brain damage.

\section{Hypoxic}

In spontaneously breathing experimental animals, including primates, the minimal level of arterial oxygen tension that does not lead to early apnoea and cardiac failure is about $20 \mathrm{~mm} \mathrm{Hg}$. At this level the cerebral vascular bed is fully dilated, the cerebral $\mathrm{A}-\mathrm{V}$ oxygen difference is reduced (due to reduced oxygen consumption and increased blood flow) but the EEG is normal. This precarious state can be disturbed by a slight fall in perfusion pressure and the immediate decline in the EEG is evidence of some reduction in cerebral blood flow. This may occur as a consequence of a period of cardiac arrhythmia. A progressive fall in heart rate and blood pressure together with slowing of respiration herald the cardio-respiratory crisis that sets the limit to the period during which the organism can tolerate this level of hypoxia. Where the circulatory failure is concerned the final bradycardia and falling blood pressure may not be a direct effect of hypoxia on the myocardium but a consequence of the stimulation of chemoreceptors in the carotid bodies or brain stem (Cross et al, 1963). As blood pressure continues to fall respiration ceases and the EEG becomes isoelectric at about the same time. Adequate resuscitation commenced soon after the 'last breath' can, as after anoxic anoxia, allow the heart to recover, spontaneous respiration to be resumed and the EEG to return to normal. Brain damage is rarely seen and only when there has been a prolonged period of cardiac impairment and an even longer period of isoelectric EEG (Brierley, Prior, Calverley, and Brownunpublished results). Brain damage in such animals consists of ischaemic neuronal alterations along the arterial boundary zones of the cerebrum and cerebellum and sometimes in the basal ganglia. This pattern of damage, indistinguishable from that seen after oligaemic hypoxia, underlines the fact that systemic hypoxaemia can only bring about brain damage through the medium of a secondary reduction in perfusion pressure.
In the human subject exposed to hypoxia not severe enough to bring about failure of respiration and the heart, the initial increase in cerebral bloodo flow may be so restricted by occlusive vascular $\Rightarrow$ disease in the arteries of the brain and/or neck that? ischaemic brain damage may ensue.

\section{Anaemic}

There is no convincing evidence that a simplesw reduction in circulating haemoglobin due to severehypochromic anaemia (iron-deficient or haemolytic) or to haemorrhage can result in brain damage. $\vec{\omega}$ Carbon monoxide intoxication remains the sole? example of anaemic hypoxia (due to the formation of stable carboxyhaemoglobin) that can be associated c $^{\infty}$ with ischaemic cell change and also with damage in $=$ white matter. The pathology in the human brain has been reviewed by Meyer (1963), Lapresle and $\vec{\infty}$ Fardeau (1966) and Brierley (1976). Ischaemic alterations may be seen in the vulnerable regions of the cortex of cerebrum and cerebellum and of the hippocampus. Necrosis in the globus pallidus is noto invariable (Meyer, 1928; Lapresle and Fardeau, 1967) and damage in white matter varies con siderably.

The presence of some element of perfusion failuse in the genesis of, at least, the cortical damage was suggested by the report of Poursines et al (1956). woman, aged 33 years, lived 26 days after attempted suicide with illuminating gas. In her brain, laminaro necrosis was distributed along arterial boundary $\frac{}{\mathbb{D}}$ zones but information concerning respiratory and $\stackrel{\varrho}{\longrightarrow}$ cardiac functions early in the survival period waso을 lacking. The case of Neubuerger and Clarke (1945), dying 13 days after carbon monoxide poisoning,

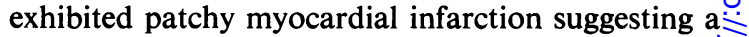
direct effect of carbon monoxide on the myocardium

Among experimental studies, that of Lewey andoDrabkin (1944) in the dog was important because, after intermittent exposures to carbon monoxide for $ᄋ$ up to 11 weeks the electrocardiograms were abnormal 2 and the brain damage was considered to be similar to응 that seen in man but was not described in detail. $\rightarrow$ Further details of electrocardiographic abnormalities were presented by Ehrich et al (1944). Recentlyñ Ginsberg et al (1974) exposed 19 Rhesus monkeys to ( 0.2 or 0.3 per cent carbon monoxide for 60 to $325^{\mathrm{N}}$ min with a carboxyhaemoglobin level of 72 to 77 per N cent throughout. Cardiac arrhythmias and somer degree of hypotension were common but the EEGo was only intermittently isoelectric. Grey matter damage (globus pallidus and hippocampus) was seen $\stackrel{\odot}{+}$ in less than a fifth of the brains while white matter 0 was abnormal in the majority. Apparently the degree $\stackrel{\vec{P}}{\overrightarrow{0}}$ of intoxication was not sufficient to produce the 
more extensive grey matter damage so often seen in the human brain.

Further confirmation of a direct effect of carbon monoxide on the heart was provided by Hodjati et al (1976) who irrigated the cerebral circulation of one dog from one carotid artery of a donor animal. A mean carboxyhaemoglobin level of 52 per cent in the donor animal led to bradycardia, hypotension and its death in 10 to $15 \mathrm{~min}$. All the recipients survived.

\section{Histotoxic}

Cyanide, the best known cause of histotoxic hypoxia, acts by inhibiting cytochrome oxidase in mitochondria while the oxygen tension and content of arterial blood remain normal. The few human cases with delayed death and evidence of brain damage have been reviewed by Brierley (1976). There was loss of neurones in cerebral cortex and cerebellum and a single case showed haemorrhagic necrosis in each globus pallidus. Hyperaemia and haemorrhages occurred in white matter.

Numerous experimental studies have suggested that cyanide, in any form and administered by any route, can damage neurones and myelin sheaths. In the first experimental study (Meyer, 1933) subcutaneous injections of potassium cyanide in dogs and rabbits produced typical ischaemic alterations in cerebral cortex, globus pallidus, hippocampus and cerebellum. White matter damage was most marked in the corpus callosum. Subsequent studies were more concerned with white matter damage because of its apparent similarity to the plaques of multiple sclerosis. However, the report of Levine and Stypulkowski (1959) was noteworthy because it suggested that grey matter damage in the rat brain after the administration of cyanide was largely due to concomitant ischaemia and hypoxic hypoxia. Brierley et al (1976) gave sodium cyanide to rats by intravenous infusion. There was full physiological monitoring in an anaesthetized group and restricted monitoring in the unanaesthetized remainder. White matter, particularly the corpus callosum, was damaged in six of 19 animals and grey matter additionally in only one. In the latter animal bradycardia, epileptic seizures and hypotension were particularly marked and it was concluded that the neuronal damage was brought about through the medium of the secondary effects of cyanide on the circulation. In a recent study in $M$ mulatta (Brierley et al, 1977) sodium cyanide was given by intravenous infusion. The effects of the infusion on respiration, heart rate, blood pressure, blood gases and the EEG were monitored in the lightly anaesthetized animals. Brain damage was seen in four of 11 animals. It involved white matter in all four but ischaemic cell change was restricted to the striatum of a single animal. In the latter there had been a period of bradycardia, hypotension and raised central venous pressure. It was concluded that in the lightly anaesthetized and spontaneously breathing Rhesus monkey, as in the rat, there is no evidence for the entity of hypoxic neuronal damage of purely histotoxic type.

\section{Hypoglycaemia}

Hypoglycaemic damage in the human brain is usually associated with irreversible coma and Meyer (1963) stated that the neuropathological findings $\therefore$. . closely resemble those which occur in other types of anoxia'. Most of the selectively vulnerable regions may be involved but with a tendency to spare the globus pallidus and cerebellum. Although hypoglycaemic coma may be associated with cardiovascular disturbances and epileptic seizures the ability of hypoglycaemia per se to produce ischaemic damage in physiologically monitored experimental primates has been demonstrated only recently.

Kahn and Myers (1971) and Myers and Kahn (1971) studied the long-term effects of insulininduced hypoglycaemia in Rhesus monkeys. Blood glucose fell to $20 \mathrm{mg} / 100 \mathrm{ml}$ after one and a half to three $h$ and was maintained at this level for four to 10 $h$ with normal blood oxygenation. In seven of 11 animals there was neuronal loss with a gliomesodermal reaction in striatum, cerebral cortex and hippocampus.

In order to define the earliest neuronal alterations due to insulin-induced hypoglycaemia, lightly anaesthetized Rhesus monkeys received insulin intravenously while EEG, EKG, heart and respiratory rates were recorded and blood gas tensions, $\mathrm{pH}$ and glucose content were measured at intervals (Meldrum et al, 1971). When blood glucose was below $20 \mathrm{mg} / 100 \mathrm{ml}$ for more than two $\mathrm{h}$ and the brains were fixed by perfusion, typical ischaemic cell change (from the stage of microvacuolation) was seen in the cortex and occasionally in striatum, hippocampus and cerebellum. Thus a major deficiency in substrate alone can produce the same type of neuronal damage as a deficiency of oxygen.

\section{Conclusion}

It will be evident from this brief review that ischaemic cell change is the cytopathological common denominator in all types of hypoxia. Nevertheless there is no pattern of its distribution specific for each category with the exception of circulatory arrest (global ischaemia) and pure hypoglycaemia after both of which ischaemic neuronal alterations may occur 
uniformly within the 'selectively vulnerable' regions of the brain. In the remaining categories of hypoxia, ie, oligaemic, anoxic, hypoxic, histotoxic and probably anaemic (carbon monoxide), an initially pure hypoxic stress in the intact and spontaneously breathing animal gives rise, sooner or later, to terminal secondary impairments of respiration and particularly of circulation. In the healthy experimental animal, however, it is only rarely that the associated period of reduced cerebral blood flow is long enough to cause brain damage but not too long to preclude recovery. In such instances, brain damage consists of a concentration of ischaemic cell change along the arterial boundary zones.

Experimental studies have shown that the terminal hypoxic cardio-respiratory deterioration or crisis consists of a slowing of respiration to the point of apnoea with a fall in blood pressure and in heart rate (but the heart may continue to beat long after the 'last breath'). There is a more or less parallel decline in EEG background activity and an isoelectric state is reached at about the time of the 'last breath'. There is no evidence to suggest that systemic hypoxia of any type can lead to brain damage unless the EEG has been isoelectric for an appreciable period. In the paralyzed and mechanically ventilated animal exposed to systemic hypoxia, initial hyperventilation as well as the 'last breath' cannot occur and the duration of hypoxia may be considerably prolonged. For this reason 'thresholds' for brain damage defined in such preparations must be applied with considerable caution to the spontaneously breathing experimental animal and to man.

There is now ample evidence to show that in the intact healthy, and spontaneously breathing animal tolerance to hypoxia is limited by the respiratory and circulatory systems and not by the intrinsic energy reserves of the brain itself. If effective resuscitation is begun soon after the 'last breath' the EEG will return and the brain will be undamaged. Thus a depression of central nervous system function up to and some time after the 'last breath' and the appearance of transiently isoelectric EEG need have no structural consequences.

The relative frequency of all degrees of ischaemic damage in the human brain after hypoxic episodes does not, however, necessarily imply a greater susceptibility of the brain itself. The existence of a single type of hypoxia in human patients is rare. It should be stressed that several types of hypoxia, each constituting a relatively mild stress can, in combination, produce brain damage. The additional factors most probably responsible for the increased extent and frequency of brain damage in man are twofold. Preexisting cardiac disease will impair the capacity to maintain a high level of blood flow through a cerebral vascular bed initially fully dilated by hypoxia. It will also impair the rapid restorations of normal cerebral blood flow after any termina $\vec{E}$ cardio-respiratory crisis. Secondly, preexisting occlu sive disease in the arteries of brain and neck and anys? impairment of the normal reactivity of the smallero cerebral vessels will further reduce cerebral bloodo flow during and after hypoxia.

In conclusion, experiments in physiologically monitored, spontaneously breathing animals canew show that hypoxia gives rise to an integrated series of responses in the respiratory and circulatory systems and in the nervous system itself. Initially these serve $\vec{\omega}$ to maintain brain function and respiration ing particular. Ultimately these compensatory cardio응 respiratory responses may fail. Experiments haves also shown that where the human brain is concerned the commonest cause of damage must be sought in. some failure of brain perfusion.

The author is greatly indebted to Dr Pamela F. Prior ${ }^{\circ}$ (The EEG Department, the London Hospital) for $\vec{C}$ many helpful suggestions and comments in thes preparation of the manuscript.

\section{References}

Barcroft, J. (1925). The Respiratory Function of the Blor Part I. Lessons from High Altitudes. Cambridge Univer Press, Cambridge.

Brierley, J. B. (1965). The influence of brain swelling, age and hypotension upon the pattern of cerebral damage ina hypoxia. Excerpta Medica International Congress Series $\frac{\circ}{\mathbb{D}}$ 100, 21-28.

Brierley, J. B. (1976). Cerebral hypoxia. In Greenfield' $\overrightarrow{\vec{\sigma}}$ Neuropathology, 3rd edition, edited by W. Blackwood and J. A. N. Corsellis, pp. 43-85. Arnold, London.

Brierley, J. B., Brown, A. W., and Calverley, J. (1976) Cyanide intoxication in the rat: physiological and neuropathological aspects. Journal of Neurology, Neurosurgert and Psychiatry, 39, 129-140.

Brierley, J. B., Brown, A. W., Excell, B. J., and Meldrum, B. S. (1969). Brain damage in the Rhesus monkey resulting. from profound arterial hypotension. I. Its nature, distri bution and general physiological correlates. Brain Research, 13, 68-100.

Brierley, J. B., and Cooper, J. E. (1962). Cerebral complica을 tions of hypotensive anaesthesia in a healthy adult. Journal of Neurosurgery and Psychiatry, 25, 24-30.

Brierley, J. B., Prior, P. F., Calverley, J., and Brown, A. W (1977). Cyanide intoxication in Macaca mulatta. Physio $\mathrm{N}$ logical and neuropathological aspects. Journal of Neuro= logical Science, 31, 133-137.

Cross, C. E., Rieben, P. A., Barron, C. I., and Salisbury, P. FN (1963). Effects of arterial hypoxia on the heart and circu- lation: an integrative study. American Journal of Physiology, 205, 963-970.

Ehrich, W. E., Bellet, S., and Lewey, F. H. (1944). Cardiac changes from CO poisoning. American Journal of Medica! Science, 208, 511-523.

Ernsting, J. (1963). Some effects of brief profound anoxia upon the central nervous system. In Selective Vulnerability of the Brain in Hypoxaemia, edited by J. P. Schadé and? 
W. M. McMenemey, pp. 41-45. Blackwell Scientific, Oxford.

Gastaut, H., Fischgold, H., and Meyer, J. S. (1961). Conclusions of the International Colloquium on Anoxia and the EEG. In Cerebral Anoxia and the Electroencephalogram, edited by H. Gastaut and J. S. Meyer, pp. 599-617. Thomas, Springfield, Illinois.

Ginsberg, M. D., Myers, R. E., and McDonagh, B. F. (1974). Experimental carbon monoxide encephalopathy in the primate. II. Clinical aspects, neuropathology, and physiologic correlation. Archives of Neurology, 30, 209-216.

Hirsch, H., Oberdörster, G., Zimmer, R., Benner, K. U., and Lang, R. (1975). The recovery of the electrocorticogram of normothermic canine brains after complete cerebral ischemia. Archiv. für Psychiatrie und Nervenkrankheiten, 221, 171-179.

Hodjati, H., Dergal, E., Montalbert, C., Goldbaum, L. R., and Absolon, K. B. (1976). Cross-circulation experiments in dogs discerning the target organ of carbon monoxide intoxication. (Abstract.) British Journal of Surgery, 63, 655.

Hoff, E. C., Grenell, R. G., and Fulton, J. F. (1945). Histopathology of the central nervous system after exposure to high altitudes, hypoglycemia and other conditions associated with central anoxia. Medicine (Baltimore), 24, 161-217.

Hossmann, K. A. and Sato, K, (1970). Recovery of neuronal function after prolonged cerebral ischemia Science, 168, 375-376.

Kahn, K. J., and Myers, R. E. (1971). Insulin-induced hypoglycaemia in the non-human primate. I. Clinical consequences. In Brain Hypoxix, edited by J. B. Brierley and B. S. Meldrum, pp. 185-194. (Clinics in Developmental Medicine 39/40, Spastics International Medical Publications). Heinemann, London.

Lapresle, J., and Fardeau, M. (1966). Les leuco-encéphalopathies de l'intoxication oxycarboneé. Étude de seize observations anatomo-cliniques. Acta Neuropathologica, 6, $327-348$.

Lapresle, J., and Fardeau, M. (1967). The central nervous system and carbon monoxide poisoning. II. Anatomical study of brain lesions following intoxication with carbon monoxide (22 cases). Progress in Brain Research, 24, 31-74.

Levine, S., and Stypulkowski, W. (1959). Effect of ischemia on cyanide encephalopathy. Neurology (Minneapolis), 9, 407-411.

Lewey, F. H., and Drabkin, D. L. (1944). Experimental chronic carbon monoxide poisoning of dogs. American Journal of Medical Science, 208, 502-511.

Meldrum, B. S., and Brierley, J. B. (1969). Brain damage in the Rhesus monkey resulting from profound arterial hypotension. II. Changes in the spontaneous and evoked electrical activity of the neocortex. Brain Research, 13, 101118.

Meldrum, B. S., and Brierley, J. B. (1971). Circulatory factors and cerebral boundary zone lesions. In Brain Hypoxia, edited by J. B. Brierley and B. S. Meldrum, pp. 20-33.
(Clinics in Developmental Medicine 39/40. Spastics International Medical Publications). Heinemann, London.

Meldrum, B. S., Horton, R. W., and Brierley, J. B. (1971). Insulin-induced hypoglycaemia in the primate: relationship between physiological changes and neuropathology. In Brain Hypoxia, edited by J. B. Brierley and B. S. Meldrum, pp. 207-224. (Clinics in Developmental Medicine 39/40. Spastics International Medical Publications). Heinemann, London.

Meyer, A. (1928). Über das Verhalten des Hemisphärenmarks bei der menschlichen Kohlenoxydvergiftung. Zeitschrift für die gesamte Neurologie und Psychiatrie, 112, 172-186.

Meyer, A. (1933). Experimentelle Vergiftungsstudien. III. Über Gehirnveränderungen bei experimenteller Blausäurevergiftung. Zeitschrift für die gesamte Neurologie und Psychiatrie, 143, 333-348.

Meyer, A. (1963). Anoxic poisons and the problems of anoxia and selective vulnerability. In Greenfield's Neuropathology, 2nd edn, edited by W. Blackwood et al., pp. 237-261. Arnold, London.

Myers, R. E., and Kahn, K. J. (1971). Insulin-induced hypoglycemia in the non-human primate. II. Long-term neuropathological consequences. In Brain Hypoxia, edited by J. B. Brierley and B. S. Meldrum, pp. 195-206. (Clinics in Developmental Medicine 39/40. Spastics International Medical Publications). Heinemann, London.

Neubuerger, K. T., and Clarke, E. R. (1945). Subacute carbon monoxide poisoning with cerebral myelinopathy and multiple myocardial necroses. Rocky Mountain Medical Journal, 42, 29-35 and 196.

Poursines, Y., Alliez, J., and Toga, M. (1956). Étude des lésions corticales d'un cas d'intoxication oxycarbonée. Revue Neurologique, 94, 731-735.

Ranck, J. B. Jr., and Windle, W. F. (1959). Brain damage in the monkey, Macaca mulatta, by asphyxia neonatorum. Experimental Neurology, 1, 130-154.

Schneider, M. (1963). Critical blood pressure in the cerebral circulation. In Selective Vulnerability of the Brain in Hypoxaemia, edited by J. P. Schadé and W. M. McMenemey, pp. 7-20. Blackwell, Oxford.

Swann, H. G., and Brucer, M. (1949). The cardiorespiratory and biochemical events during rapid anoxic death. I. Fulminating anoxia. Texas Reports on Biology and Medicine, 7, 511-538.

Symon, L., and Brierley, J. B. (1976). Morphological changes in cerebral blood vessels in chronic ischemic infarction: flow correlation obtained by the hydrogen clearance method. In The Cerebral Vessel Wall, edited by J. CervósNavarro, et al., pp. 165-174. Raven Press, New York.

Zülch, K. J., and Behrend, R. C. H. (1961). The pathogenesis and topography of anoxia, hypoxia and ischemia of the brain in man. In Cerebral Anoxia and the Electroencephalogram, edited by H. Gastaut and J. S. Meyer, pp. 144-163. Thomas, Springfield, Illinois. 